\title{
A Block-by-Block Method for Volterra Integro-Differential Equations With Weakly-Singular Kernel
}

\author{
By Athena Makroglou*
}

\begin{abstract}
The theory of a block-by-block method for solving Volterra integro-differential equations with continuous kernels (see Makroglou [4], [5] is adapted to Volterra integrodifferential equations with weakly-singular kernels, and a rate of convergence is given.
\end{abstract}

1. Introduction. Consider the nonlinear Volterra integro-differential equation

$$
y^{\prime}(x)=G\left(x, y(x), \int_{0}^{x} K(x, t, y(t))\right) d t \quad(x>0),
$$

given $y(0)$, written in the form,

$$
\begin{aligned}
& y(x)=\int_{0}^{x} G(s, y(s), z(s)) d s+y(0) \quad(x>0), \\
& z(x)=\int_{0}^{x} K(x, t, y(t)) d t \quad(x>0),
\end{aligned}
$$

with

$$
\begin{gathered}
K(x, s, y(s)) \equiv K(x, s) y(s), \\
K(x, s)=1 /|x-s|^{\alpha}, \quad 0<\alpha<1,0<s<x<X .
\end{gathered}
$$

For the discretization of the equation (1.3), we shall use a product integration technique in such a way that when the method is used for solving examples with $K(x, s, y(s))=H(x, s, y(s)) /|x-s|^{\alpha}$ it will not require the evaluation of $H(x, s, y(s))$ for $s>x$, where it might, for example, not be defined (see Section 2). Product integration techniques have been used for the solution of weakly-singular integral equations; see for example Linz [3], Weiss [6], de Hoog and Weiss [2], Baker [1].

For the discretization of Eq. (1.2) we shall use Eqs. (2.3) in Makroglou [5] and produce a scheme which we called a generalized block-by-block method after Weiss, scheme GC, though it is a new method for integro-differential equations, see Section 3 below, originated in [4]. (' $G$ ' stands for 'Generalized' and ' $C$ ' is kept here in agreement with the notation used in [4] where it meant the third of the $G$ schemes GA, GB, GC.)

A rate of convergence of the scheme is given in Section 4.

For use in the discussion to follow, we define $x_{m, j}=m h+u_{j} h, x_{m, j, k}=m h+$ $u_{j} u_{k} h, j, k=0,1, \ldots, p ; m=0,1, \ldots, N-1$, where $N, p$ integers, $h>0$ so that $N h=X$ and $0<u_{0}<u_{1}<\cdots<u_{p}=1$. We also assume the preliminaries and definitions given in Makroglou [5].

Received January 29, 1980; revised August 14, 1980.

AMS (MOS) subject classifications (1970). Primary 65R05; Secondary 65D05, 65D30.

*Formerly of the Department of Mathematics, University of Manchester, England. 
2. Discretization of Eq. (1.3). Consider the equation (1.3) with $K(x, s, y(s))$ as in (1.4), that is the equation,

$$
z(x)=\int_{0}^{x} K(x, t) y(t) d t
$$

where $K(x, t)$ is given by (1.4). Discretizing at the points $x_{m, j}$ we have

$$
z\left(x_{m, j}\right)=\sum_{i=0}^{m-1} \int_{i h}^{(i+1) h} K\left(x_{m, j}, s\right) y(s) d s+\int_{m h}^{x_{m, j}} K\left(x_{m, j}, s\right) y(s) d s,
$$

or

$$
\begin{aligned}
z\left(x_{m, j}\right)= & h \sum_{i=0}^{m-1} \int_{0}^{1} K\left(x_{m, j}, i h+h t\right) y(i h+h t) d t \\
& +h u_{j} \int_{0}^{1} K\left(x_{m, j}, m h+u_{j} h t\right) y\left(m h+u_{j} h t\right) d t
\end{aligned}
$$

We now use the approximations

$$
\begin{aligned}
y(i h+h t) & \simeq \sum_{k=0}^{p} L_{k}(t) y\left(x_{i, k}\right), \\
y\left(m h+h u_{j} t\right) & \simeq \sum_{k=0}^{p} L_{k}(t) y\left(m h+u_{j} u_{k} h\right) \\
& \simeq \sum_{k=0}^{p} L_{k}(t) \sum_{r=0}^{p} L_{r}\left(u_{j} u_{k}\right) y\left(x_{m, r}\right),
\end{aligned}
$$

where $L_{k}(t)$ are the Lagrangian coefficients, giving

$$
\begin{aligned}
z_{m, j}= & h u_{j} \sum_{r=0}^{p} \sum_{k=0}^{p} V^{(m)}(m, j, k) L_{r}\left(u_{j} u_{k}\right) y_{m, r} \\
& +h \sum_{i=0}^{m-1} \sum_{k=0}^{p} V^{(m)}(i, j, k) y_{i, k},
\end{aligned}
$$

$m=0,1, \ldots, N-1 ; j=0,1, \ldots, p,\left(j=1,2, \ldots, p\right.$, if $\left.u_{0}=0\right)$, where we have put

$$
V^{(m)}(i, j, k)=\int_{0}^{1} K\left(x_{m, j}, i h+u h t\right) L_{k}(t) d t
$$

with

$$
\begin{array}{ll}
u=u_{j} & \text { if } i=m, \\
u=1 & \text { if } i=0,1, \ldots, m-1 .
\end{array}
$$

2.1. Estimation of the Coefficients $V^{(m)}(i, j, k)$. Using the kernel (1.4) in (2.7), we obtain

$$
V^{(m)}(i, j, k)=\int_{0}^{1} \frac{\prod_{q=0 ; q \neq k}^{p}\left(t-u_{q}\right)}{|l-t|^{\alpha}} d t /\left(u^{\alpha} h^{\alpha} D(k)\right)
$$

where

$$
D(k)=\prod_{q=0 ; q \neq k}^{p}\left(u_{k}-u_{q}\right)
$$


and

$$
\begin{array}{ll}
l=m+u_{j}-i & \text { for } i=0,1, \ldots, m-1, \\
l=1 & \text { for } i=m,
\end{array}
$$

or

$$
V^{(m)}(i, j, k)=(-1)^{p+1} \int_{l^{\alpha}}^{(l-1)^{\alpha}} \prod_{q=1}^{p}\left(t^{1 / \alpha}-a_{q}\right) t^{1 / \alpha-2} d t /\left(\alpha u^{\alpha} h^{\alpha} D(k)\right)
$$

where

$$
\begin{array}{ll}
a_{q+1}=l-u_{q}, & q=0,1, \ldots, k-1, \\
a_{q}=l-u_{q}, & q=k+1, \ldots, p .
\end{array}
$$

The product $\Pi_{q=1}^{p}\left(t^{1 / \alpha}-a_{q}\right)$ in (2.12) can be written as

$$
\prod_{q=1}^{p}\left(t^{1 / \alpha}-a_{q}\right)=c_{0}\left(t^{1 / \alpha}\right)^{p}+c_{1}\left(t^{1 / \alpha}\right)^{p-1}+\cdots+c_{p}
$$

where, with $S_{m}=a_{1}^{m}+a_{2}^{m}+\cdots+a_{p}^{m}$, we have

$$
\begin{aligned}
& c_{0}=1, \\
& c_{1}=-S_{1}, \\
& c_{j}=-\left(S_{j}+c_{1} S_{j-1}+c_{2} S_{j-2}+\cdots+c_{j-1} S_{1}\right) / j, \quad j=2,3, \ldots
\end{aligned}
$$

Substituting (2.14) in (2.12) and integrating, we find

$$
V^{(m)}(i, j, k)=\frac{(-1)^{p+1}}{u^{\alpha} h^{\alpha} D(k)} \sum_{r=0}^{p} c_{p-r} \frac{\left\{(l-1)^{r-\alpha+1}-l^{r-\alpha+1}\right\}}{r-\alpha+1},
$$

$i=0,1, \ldots, m ; k=0,1, \ldots, p ; j=1, \ldots, p$ if $u_{0}=0, j=0,1, \ldots, p$ if $u_{0} \neq 0$.

3. Statement of the Method. According to the illustration given in the introduction, the approximate equations for scheme $\mathrm{GC}$ are

$$
\begin{aligned}
y_{m, j}= & h \sum_{k=0}^{p} w_{k}^{j} G\left(x_{m, k}, y_{m, k}, z_{m, k}\right) \\
& +h \sum_{i=0}^{m-1} \sum_{k=0}^{p} w_{k} G\left(x_{i, k}, y_{i, k}, z_{i, k}\right)+y(0), \\
z_{m, j}= & h u_{j} \sum_{r=0}^{p} \sum_{k=0}^{p} V^{(m)}(m, j, k) L_{r}\left(u_{j} u_{k}\right) y_{m, r} \\
& +h \sum_{i=0}^{m-1} \sum_{k=0}^{p} V^{(m)}(i, j, k) y_{i, k},
\end{aligned}
$$

$m=0,1, \ldots, N-1 ; j=0,1, \ldots, p,\left(j=1,2, \ldots, p\right.$ if $\left.u_{0}=0\right)$, where

$$
\begin{gathered}
w_{k}^{j}=\int_{0}^{u_{j}} L_{k}(x) d x, \\
w_{k}=w_{k}^{p}=\int_{0}^{1} L_{k}(x) d x,
\end{gathered}
$$




$$
L_{k}(x)=\prod_{j=0 ; j \neq k}^{p}\left(x-u_{j}\right) /\left(u_{k}-u_{j}\right),
$$

and $V^{(m)}(i, j, k)$ are given by (2.16).

Equations (3.1)-(3.2) constitute a system of $2 p+2\left(2 p\right.$ if $\left.u_{0}=0\right)$ in general nonlinear equations for $y_{m, 0}, y_{m, 1}, \ldots, y_{m, p} ; z_{m, 0}, z_{m, 1}, \ldots, z_{m, p}$.

4. Convergence. For the complete convergence proofs we refer to [4]. There, we started by obtaining an asymptotic expansion for the error $\varepsilon_{m} \equiv \max _{0<j<p}\left|\varepsilon_{m, j}\right|$, $\varepsilon_{m, j} \equiv z\left(x_{m, j}\right)-z_{m, j}$ in the approximations (3.2). In doing this, the work in [2] was of great help. Having obtained this expansion, one can then obtain a bound on $\mathbf{s}_{m}=\left[e_{m}, \varepsilon_{m}\right]^{T}$ along the lines of the convergence proof given in [5]. The convergence result obtained is given as Theorem 1 below.

THEOREM 1. Let

(i) $g(x) \in P_{v}$ (see preliminaries in [5]),

(ii) $y(x)$ is $p+2$ times continuously differentiable on $0<x<X$,

(iii) $G(x, y, z)$ be $p+v+2$ times continuously differentiable with respect to $x, y, z$, respectively, on $0 \leqslant x \leqslant X,|y| \leqslant \bar{y},|z| \leqslant \bar{z}$ where $\bar{y}=\max _{0<x<x}|y(x)|$ and $\bar{z}=\max _{0<x<x}|z(x)|$. Then, there are constants $C_{1}, C_{2}, C_{3}, C_{4}, C_{5}$ such that

$$
\left\|\mathrm{s}_{m}\right\|_{\infty} \leqslant C_{5} h^{p+1} \text { if } v=0
$$

$$
\left\|\mathbf{s}_{m}\right\|_{\infty} \leqslant\left\{\begin{array}{ll}
C_{1} h^{p+2} & \text { (1) } \\
C_{2} h^{p+2-\alpha * *} & \text { (2) }
\end{array} \text { if } v>0\right.
$$

$m=1,2, \ldots, N-1$, and

$$
\left\|\mathrm{s}_{0}\right\|_{\infty} \leqslant\left\{\begin{array}{l}
C_{3} h^{p+2} \\
C_{4} h^{p+2-\alpha}
\end{array}\right.
$$

and the inequalities occur with (1) or (2) according to where the maximum occurs when considering $\|\cdot\|_{\infty}$.

Some numerical results obtained by testing scheme GC on a linear and a nonlinear example for both $u_{0}=0, u_{0} \neq 0$ are displayed in [4] (see [4, Examples 3, 4, p. 97 ; pp. 152, 153, 157, 158]). Order of convergence at least $O\left(h^{p+1}\right)$ was verified.

Acknowledgement. This work forms part of the author's Ph.D. thesis written at the University of Manchester under the supervision of Dr. C. T. H. Baker and Dr. Ian Gladwell (for six months) both of whom the author wishes to thank, from here too, for their valuable advice, encouragement, and criticism.

The M.Sc. and Ph.D. work of the author was supported mainly (for 34 months) by the Greek State Scholarships Foundation.

Statistical Unit

Athens University

Panepistemiopolis

Athens-621, Greece

**The result (2) in (4.1) is changed here to $C_{2} h^{p+2-\alpha}$ from $C_{2} h^{p+1}$ in [4]. This because in [4, p. 201, Eq. III-1.108] we have $\int_{0}^{1} g(t) P_{0}(t) d t=0$ for $g \in P_{v(>0)}$. 
1. C. T. H. BAKER, The Numerical Treatment of Integral Equations, Clarendon Press, Oxford, 1977.

2. F. DE Hoog \& R. WeIss, “Asymptotic expansions for product integration," Math. Comp., v. 27, 1973, pp. 295-306.

3. P. LnN, "Numerical methods for Volterra integral equations with singular kernels," SIAM J. Numer. Anal., v. 6, 1969, pp. 365-374. MR 41 \#4850.

4. A. Makroglou, Numerical Solution of Volterra Integro-Differential Equations, Ph.D. thesis, Univ. of Manchester, U.K., Feb. 1977.

5. A. MAKroglou, "Convergence of a block-by-block method for nonlilnear Volterra integrodifferential equations," Math. Comp., v. 35, 1980, pp. 783-796.

6. R. WeIss, Numerical Procedures for Volterra Integral Equations, Ph.D. thesis, Computer Centre, Australian National University, Canberra, 1972. 